\title{
Mobile applications for postoperative monitoring after discharge
}

\author{
John L. Semple MD MSc, Kathleen A. Armstrong MD MSc
}

Cite as: CMAJ 2017 January 9;189:E22-4. doi: 10.1503/cmaj.160195

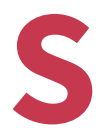
martphone technology appears to be the next phase of development in telemedicine. With the increased focus on patient-centred care, mobile technology is being used to connect patients and their health care teams. Numerous mobile applications are available that can assist with both surgical and medical interventions, as well as with behavioural changes in smoking cessation and weight management. ${ }^{1}$ Mobile applications can help patients self-manage chronic conditions such as diabetes and congestive heart failure by providing an opportunity to enter their own data and symptoms on their smartphone that can be relayed in real time to the care team. ${ }^{1}$ Health care providers can use these patient-generated data to act on abnormal trends in symptoms. Mobile applications can be used not only as a technology adjunct for point-of-care and as a diagnostic tool, ${ }^{1,2}$ but also to send medication and appointment reminders. ${ }^{1}$

In surgical care, there is a growing trend to reduce the total length of stay in hospital while minimizing readmissions and increasing patient engagement in self-care. However, expedited discharge with the patient recovering at home may result in a lack of outcome data that could become a barrier to quality improvement. To address this challenge, mobile applications have been deployed to monitor and support discharge transition and recovery after surgery, both in urban and rural settings.,3

The first 30 days after discharge from hospital is a pivotal period for postoperative patients because this is when most complications occur, leading to unexpected visits to the emergency department and readmissions. ${ }^{3}$ Over the past few years, surgical clinics have attempted to capitalize on new features of mobile phones, such as basic texting or photographic capabilities, to

\section{KEY POINTS}

- In the era of patient-centred care, mobile technology is being used to connect patients and their health care teams.

- In surgical care, use of remote monitoring may allow improved transition care and greater insight regarding the development of postoperative complications and readmissions.

- Mobile applications must address best practices and regulatory standards with regard to privacy, security and patient confidentiality.

- Deployment of mobile applications in postoperative follow-up should remain affordable and not widen existing disparities of access and cost in health care.

monitor patients after discharge from hospital in different types of surgical procedures, including ophthalmologic, otolaryngologic, plastic, orthopedic and gastrointestinal surgery. ${ }^{3-5}$

\section{How are mobile applications used postsurgically?}

Mobile applications have been used during the postoperative period for three general purposes: replacing scheduled followup visits, ongoing monitoring and the remote identification of complications as they appear. ${ }^{2-4,6}$ Examples include patients communicating their recovery symptoms by text messaging or responding to validated questionnaires that contain quality-ofrecovery indicators such as degree of nausea and/or vomiting (Box 1, Figure 1). More specific quantitative data can be col-

\section{Box 1: Use of a postsurgical application at the Women's College Hospital}

At the Women's College Hospital in Toronto, Ontario, we are conducting a randomized controlled trial, funded by the Canadian Institutes of Health Research, using a mobile application (created by QoC Health, Toronto) to conduct postoperative follow-up care and to monitor for postoperative complications after the patient has returned home. The Women's College Hospital is an ambulatory facility with no inpatient beds. Although the hospital continues to offer complex surgical procedures, most patients go home the same day. Patients undergoing breast reconstruction after surgery for breast cancer have an average travelling distance of $75 \mathrm{~km}$ for a return follow-up visit.
During a preadmission clinic visit, patients are offered an option to use the application. The application can be downloaded onto patients' personal smartphone or onto a device loaned to them for the 30-day period if they do not own a smartphone. Once the patients have been discharged, they answer a series of questions using a touch-screen interface on their smartphone related to their quality of recovery (Figure 1) and take pictures of their surgical incision site daily. This information is relayed to a secure cloud server, where it is available on a dashboard for viewing by their health care team, which consists of the surgeon, advanced practice nurse and surgical resident (Figure 2). 
lected using text messages or touchscreen visual analogue features on smartphones for daily drain outputs and pain profiles. ${ }^{2,6}$ Other types of data that can be collected include alerts for responses in the extreme range that could potentially lead to readmission, including uncontrolled pain., ${ }^{2,6}$

More complex multimodal monitoring of symptoms such as blood pressure and oxygen saturation have been captured by using wearable devices and Bluetooth-compatible features. ${ }^{7}$ Digital pho-
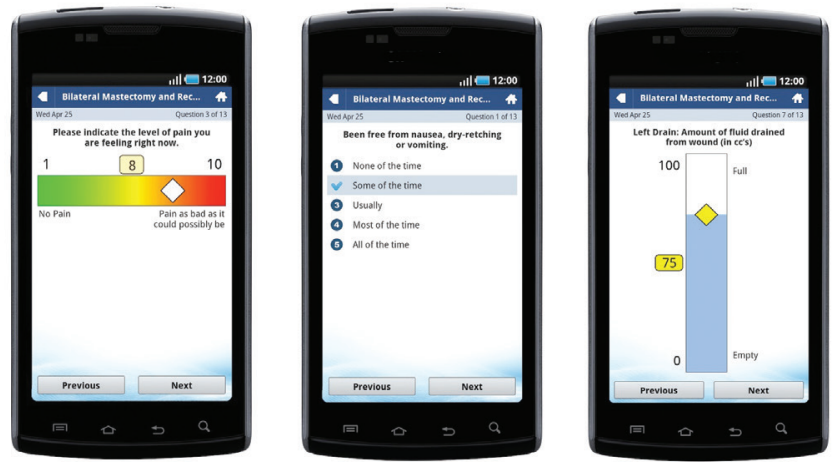

Figure 1: Examples of the touch-screen interface of the patient portal, including a visual analogue scale for pain, an example of the questions on postoperative nausea and the visual analogue scale for fluid in the postoperative surgical drains. ( John L. Semple, Sarah Sharpe, M. Lucas Murnaghan, John Theodoropoulos, Kelly A. Metcalfe. Originally published in JMIR mHealth and uHealth (http://mhealth.jmir.org). ${ }^{6}$ tographic features on smartphones can be used to monitor changes in the surgical site. These images can be evaluated using store-andforward technology, in which information is transmitted to a secure cloud server for review by the health care provider ${ }^{6}$ (Figure 2).

\section{What are the benefits?}

Mobile applications - and telemedicine in general - have been shown to improve the care transition of postoperative patients from institution to home. ${ }^{7}$ The overall benefits of postoperative monitoring using mobile applications can be divided into those affecting patients and those affecting the health care system. Patients have realized cost savings, particularly in round-trip kilometers saved, reduced time off work for family members who accompany the patient on return clinic visits and elimination of nights away from home in hotels. ${ }^{6,7}$ Patients have expressed satisfaction with the convenience associated with using mobile applications and telemedicine in postoperative follow-up..$^{6-8}$

Although no study has shown improved clinical outcomes with use of mobile applications for postoperative monitoring, there have been system savings reported, both in the United States and Canada. The availability of additional clinic spots for new patients as a result of monitoring patients at home has been reported in areas with limited access to specialty care and documented long wait times. ${ }^{6-9,10,11}$ One telemedicine follow-up study, which monitored patients at home after open hernia repair, showed that 110 clinic spots became available during the 10 -month study period. ${ }^{11}$

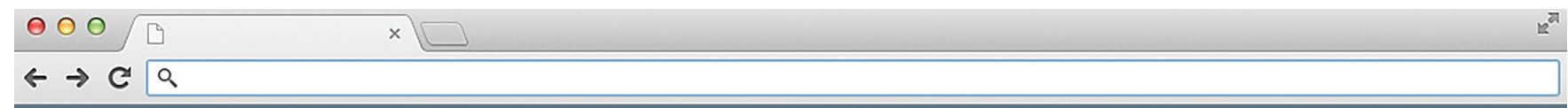

Q Home Reports > Patient Procedure List > Patient Monitoring

Photo Stream

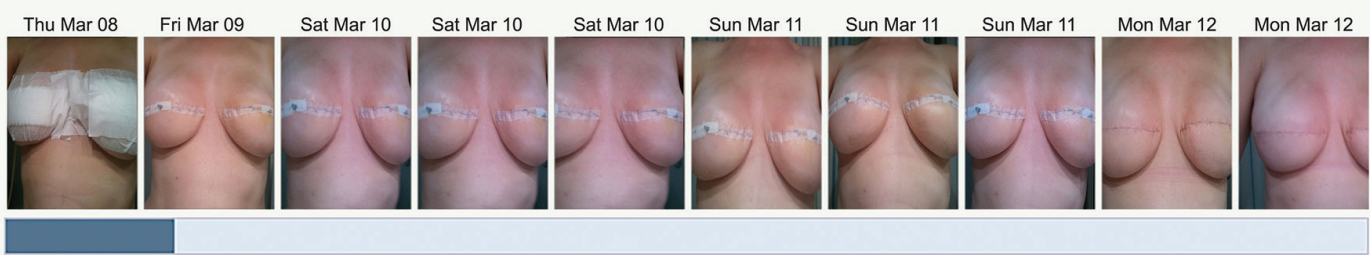

Feedback Navigator

\begin{tabular}{ll|l|l|l|l|l|l|l|} 
(4) Wed Apr 11 & Sat Apr 14 & Sun Apr 15 & Tue Apr 17 & Wed Apr 18 & Sun Apr 22 & Mon Apr 23 & Fri Apr 28 \\
\hline
\end{tabular}

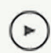

You can sort by clicking on the column titles, hold shift to select multiple columns

\begin{tabular}{|c|c|c|c|c|c|}
\hline Q\# $\triangle$ & Question & Option Text & Value $\Rightarrow$ & SparkLine & Entry Date \\
\hline 1 & Been free from nausea, dry-retching or vomiting. & Usually & 3 & $\rho$ Pr. & Apr 232013 03:03 pm \\
\hline \multirow[t]{2}{*}{2} & Been free from experiencing severe pain or constant moderate pain. & Most of the time & 2 & 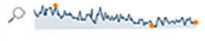 & Apr 232013 03:05 pm \\
\hline & Please indicate the level of pain you are feeling right now. & & 8 & OP & Apr 232013 03:06 pm \\
\hline 4 & Been able to breathe easily. & All of the time & 1 & אמיוניו & Apr 232013 03:08 pm \\
\hline
\end{tabular}

Figure 2: Image of the surgeon's dashboard. The surgeon or care provider can view the patient's quality of recovery on one screen. The photos show the patient's own pictures of the surgical site with the date. The questions are listed with the patient's responses. Question \#3 is highlighted because of the abnormally high pain score entered by the patient. The sparkline represents every score as a point of data on that particular indicator since the start of the monitoring. The pictures can be enlarged by tapping on them. C John L. Semple, Sarah Sharpe, M. Lucas Murnaghan, John Theodoropoulos, Kelly A. Metcalfe. Originally published in JMIR mHealth and uHealth (http://mhealth.jmir.org). ${ }^{6}$ 


\section{What are the possible harms?}

Despite the reported benefits of postoperative monitoring with mobile applications, there are several issues that should be addressed as this innovation finds greater adoption. Not all mobile applications adhere to best practices in privacy, security and patient confidentiality. ${ }^{7}$ Best practices and emerging telemedicine regulatory frameworks should be considered in the design, development and deployment of the mobile applications.

Currently, there is an absence of professional and legal guidelines regarding the safe use of mobile camera phones in a clinical setting. ${ }^{7}$ The Health Insurance Portability and Accountability Act, the federal Personal Information Protection and Electronic Documents Act, and province-specific personal health information protection acts that define the rules of engagement and hold organizations accountable for the protection of personal information should be considered in use of these devices. Minimal use of patient identifiers should be adopted and appropriate encryption should be a feature of any health mobile application that stores and manages patient data. This would include encryption for in-transit data, data stored in servers or in the "cloud," and potential ability to wipe data remotely from a lost phone if necessary. ${ }^{9}$

Additional limitations include concerns about physician compensation for care delivered by new technologies, such as mobile applications, and the possibility that access to these technologies may be limited to patients who can afford it or are able to understand how to use it. Finally, whereas large academic health centres may be able to adopt mobile application programs, smaller, more remote centres may have less access to the resources required to keep pace with such rapid change. ${ }^{12}$

\section{What is the evidence so far?}

The use of telephone follow-up using standardized questionnaires after ambulatory surgery is a well-established practice at many institutions. ${ }^{7}$ With the proliferation of mobile technology, several studies have investigated use of mobile applications for effective follow-up in the postoperative period, deploying different program designs in a variety of different surgical populations. ${ }^{2-7}$

Current evidence supports substantial time, travel and cost savings to patients and high satisfaction with the convenience of postoperative monitoring protocols using mobile applications. ${ }^{6-8}$ Provider satisfaction with the concept of these programs is also high. ${ }^{6-8}$ There is some early evidence for cost savings from the perspective of health care systems, but larger, long-term studies are required to provide definitive evidence..$^{7-9,11}$

\section{What can we expect in the future?}

Mobile applications are increasingly being used in a variety of procedure-based initiatives for surgical patients in the postoperative period. Protocols based on smartphone applications will continue to provide improved transition of care for surgical patients in both ambulatory and inpatient settings, as well as more comprehensive postoperative monitoring with both objective and patientgenerated data analytics. It is likely that further evidence will show the effect of postoperative monitoring via mobile applications on containment of costs, both from the perspective of patients and the health care system. ${ }^{13}$

\section{References}

1. Mosa ASM, Yoo I, Sheets L. A systematic review of healthcare applications for smartphones. BMC Med Inform Decis Mak 2012;12:67.

2. Martínez-Ramos C, Cerdán MT, López RS. Mobile phone-based telemedicine system for the home follow-up of patients undergoing ambulatory surgery. Telemed J E Health 2009;15:531-7.

3. Kazaure HS, Roman SA, Sosa JA. Association of postdischarge complications with reoperation and mortality in general surgery. Arch Surg 2012;147:1000-7.

4. Sanger PC, Hartzler A, Han SM, et al. Patient perspectives on post-discharge surgical site infections: towards a patient-centered mobile health solution. PLoS One 2014;9:e114016.

5. Armstrong KA, Semple JL, Coyte PC. Replacing ambulatory surgical follow-up visits with mobile app home monitoring: modeling cost-effective scenarios. J Med Internet Res 2014;16:e213.

6. Semple JL, Sharpe S, Murnaghan ML, et al. Using a mobile app for monitoring post-operative quality of recovery of patients at home: a feasibility study. JMIR Mhealth Uhealth 2015;3:e18.

7. Gunter RL, Chouinard S, Fernandes-Taylor S, et al. Current use of telemedicine for post-discharge surgical care: a systematic review. J Am Coll Surg 2016;222: 915-27.

8. Armstrong KA, Coyte PC, Bhatia RS. The effect of mobile app home monitoring on the number of in-person visits following ambulatory surgery: protocol for a randomized controlled trial. JMIR Res Protoc 2015;4:e65.

9. Health care in Canada, 2012: a focus on wait times. Ottawa: Canadian Institute for Health Information; 2012. Available: https://secure.cihi.ca/free_products/ HCIC2012-FullReport-ENweb.pdf (accessed 2016 Sept. 25).

10. Cloud computing in health: white paper. Toronto, Montréal, Halifax, Vancouver: Canada Health Infoway; 2012. Available: www.infoway-inforoute.ca/en/component/ edocman/545-cloud-computing-in-health-white-paper-full/view-document?ltemid $=101$ (accessed 2015 Sept. 3).

11. Hwa K, Wren SM. Telehealth follow-up in lieu of postoperative clinic visit for ambulatory surgery: results of a pilot program. JAMA Surg 2013;148:823-7.

12. Gray RT, Sut MK, Badger SA, et al. Post-operative telephone review is costeffective and acceptable to patients. Ulster Med J 2010;79:76-9.

13. Kahn JM. Virtual visits - confronting the challenges of telemedicine. N Engl J Med 2015;372:1684-5.

Competing interests: John Semple and Kathleen Armstrong have received funding from the Canadian Institutes of Health Research e-Health Innovations Catalyst grants and Strategy for PatientOriented Research networks. This includes support for a randomized controlled trial currently underway at the Women's College Hospital comparing conventional in-person and mobile-application follow-up care. John Semple holds shares in QoC Health.

This article has been peer reviewed.

The authors have obtained patient consent.

Affiliations: Division of Plastic Surgery (Semple, Armstrong), Women's College Hospital; Department of Surgery (Semple), University of Toronto, Toronto, Ont.

Contributors: John Semple contributed substantially to conception, design and drafting of the manuscript, along with the interpretation of data. Kathleen Armstrong was responsible for acquisition and analysis of data, and contributed to drafting the manuscript. Both authors gave final approval of the version to be published and agreed to act as guarantors of the work.

Correspondence to: John Semple, john.semple@wchospital.ca 